\title{
VALIDAÇÃO DE DADOS ANEMOMÉTRICOS A PARTIR DE PADRÕES ESPAÇO-TEMPORAIS DE VENTO EM SUPERFÍCIE
}

\author{
Jonathan Amanajás ${ }^{1,2}$, Samira Santos ${ }^{1,2}$, Daniel Faro ${ }^{1}$, Célia Braga ${ }^{2}$ \\ 1 Centro de Tecnologias do Gás \& Energias Renováveis \\ 2 Universidade Federal de Campina Grande \\ jonathan.amanajas@hotmail.com
}

\section{RESUMO}

Diferentes fatores podem comprometer a confiabilidade de dados anemométricos medidos em campo, podendo alterar as medições, tornando-as incorretas. A disponibilização de dados de torres anemométricas requer que estes sejam submetidos a um rigoroso processo de controle de qualidade, cujo objetivo é identificar dados inconsistentes ou suspeitos. O desenvolvimento de um conjunto de critérios de validação destes dados é elemento crítico de sua avaliação. Neste estudo foi aplicado a técnica de análise fatorial em componentes principais (ACP) para avaliar padrões espaço-temporais do campo velocidade do vento em superfície sobre o norte do Nordeste brasileiro, cujo objetivo foi particularizar a variabilidade do regime de vento sobre a região, tornando os critérios de validação mais precisos durante a análise destes. Os resultados mostram que as maiores contribuições do regime eólico ocorrem sobre o litoral Cearense e Potiguar durante o semestre setembrofevereiro, período menos chuvoso da região. A partir destes resultados foi possível regionalizar os valores fixados nos algoritmos de validação das séries de dados observados, uma vez que passou-se a conhecer seu comportamento sazonal e distribuição espacial.

Palavras-chave: Análise Multivariada,Validação de dados, Regionalização. 


\begin{abstract}
Different factors may compromise the reliability of data anemometer measured in the field and can change the measurements, making them inaccurate. The availability of data anemometric towers requires that they be subjected to a rigorous quality control process, whose goal is to identify suspicious or inconsistent data. Developing a set of criteria for validation of these data is a critical element of their evaluation. This study applied the technique of principal component factor analysis (PCA) to evaluate spatial and temporal patterns of the velocity field in the surface winds over the north of the Brazilian Northeast, whose goal was to particularize the variability of wind regime on the region, making validation criteria for more accurate analysis of these. The results show that the major contributions of the wind regime occur on the coast of Ceará and Rio Grande do Norte during the semester from September to February, less rainy season in the region. From these results it was possible to localize the values set in the algorithms validation data sets observed, since it moved to meet their seasonal behavior and spatial distribution.
\end{abstract}

Keywords: Multivariate Analysis, Data validation, Regionalization.

\title{
INTRODUÇÃO
}

Diversos fatores podem comprometer a confiabilidade de dados medidos em campo por equipamentos automáticos. Por isso, antes de disponibilizar os dados medidos em torres anemométricas, estes são submetidos a um processo de controle de qualidade, cujo objetivo é identificar e corrigir possíveis erros de forma rápida, minimizando seu impacto sobre o resultado dos estudos.

A análise de dados deve ser baseada tanto no conhecimento de erros técnicos como na variação de valores normais das observações que estão distantes das restantes ou são inconsistentes com as mesmas que são habitualmente designadas como espúrias. No entanto, os métodos de controle de qualidade recomendados referem-se apenas a análises de dados em tempo real e para curtos intervalos de tempo e, geralmente, não incluem testes baseados em análises de séries temporais, tais como métodos de homogeneidade e análises de 
variância, que, por exemplo, podem ser utilizados para verificar se há significativa sazonalidade nos dados medidos.

Assim, este estudo teve como propósito determinar objetivamente os padrões espaçotemporais da velocidade do vento sobre o norte do Nordeste brasileiro, a fim de caracterizar sua variabilidade sobre a região, tornando os ranges adotados nos critérios de validação mais precisos e partilarizados, colaborando diretamente no controle da qualidade dos dados coletados.

\section{MATERIAL E MÉTODOS}

A área de estudo compreende os Estados do Ceará e Rio Grande do Norte, no norte do Nordeste brasileiro. Foram analisados dados mensais de velocidade do vento $(\mathrm{m} / \mathrm{s})$ a 10 metros de altura, de 19 estações meteorológicas do Instituto Nacional de Meteorologia, cujas séries temporais têm em média 35 anos de observações.

Os padrões dominantes da variabilidade do regime de vento sobre o norte do Nordeste brasileiro foram determinados através do método de Análise Fatorial em Componentes Principais (ACP). Neste estudo, utilizou-se a ACP em Modo-T, aplicado para criar agrupamentos de variáveis e classificar seus campos atmosféricos espaciais, quantificando sua frequência e mostrando os períodos de tempo neles dominantes, ou seja, obter a evolução dos principais modos dominantes de circulação que permitam analisar a trajetória e comportamento dos sistemas sinóticos sobre determinadas áreas [1-2].

\section{RESULTADOS E DISCUSSÕES}

Os dois primeiros fatores temporais que explicam 95,34\% da variância total dos dados mensais de vento foram retidos. O primeiro fator temporal explica 48,18\% da variância total dos dados, com maiores correlações positivas $(>0,7)$ nos meses de setembro a fevereiro. A Figura 1a corresponde ao padrão espacial do primeiro fator temporal e apresenta as maiores contribuições da velocidade do vento sobre o litoral cearense e potiguar. Sugere-se que o regime eólico associado a este padrão está relacionado com a atuação e deslocamento da Zona de Convergência Intertropical (ZCIT) mais ao norte de sua climatologia (cerca de $14^{\circ} \mathrm{N}$ em agosto/setembro), o que favorece a intensificação das circulações de brisas marítimas e 
terrestres. Na Figura 1b, identifica-se uma faixa zonal em torno de $5^{\circ} \mathrm{N}$ sobre o Atlântico e o continente adjacente, em que o limiar de radiação de onda longa (ROL) $\leq 240 \mathrm{Wm}^{-2}$, capaz de separar as áreas convectivas das áreas não convectivas [3], indica a posição da banda de nebulosidade profunda associada à ZCIT. Desta forma, espera-se que as maiores variações diárias, bem como horárias, da velocidade do vento, sobre esta região de maiores escores fatoriais, ocorram entre os meses de setembro a fevereiro.

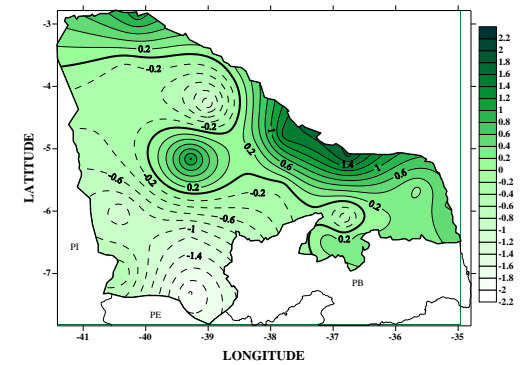

(a)

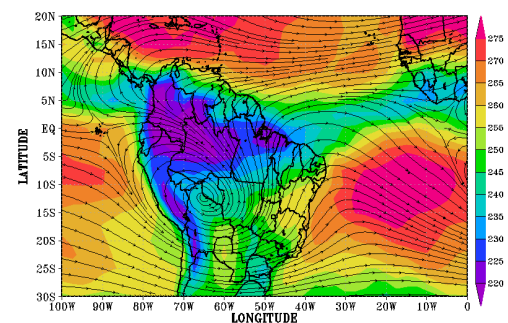

(b)

Figura 1 - (a) Padrão espacial do primeiro fator temporal da velocidade do vento e (b) climatologia semestral (setembro a fevereiro) de radiação de ondas longas $\left(\mathrm{Wm}^{-2}\right)$ e vento em $200 \mathrm{hPa}$ (linhas de corrente, ms $^{-1}$ ).

O segundo fator temporal, que explica 47,16\% da variância total dos dados, tem maiores correlações positivas $(>0,7)$ nos meses de março a agosto. $\mathrm{O}$ padrão espacial associado ao segundo fator temporal apresenta as maiores contribuições da velocidade do vento sobre o litoral norte e leste do Rio Grande do Norte (Figura 2a), também relacionado ao deslocamento da ZCIT mais ao norte de sua climatologia (Figura 2b). Observou-se que o Estado do Ceará apresenta maior variabilidade espaço-temporal do regime de vento, enquanto o Estado do Rio Grande do Norte maior homogeneidade espacial, apresentando contribuições significativas de velocidade do vento ao longo de todo o ano sobre sua região litorânea.

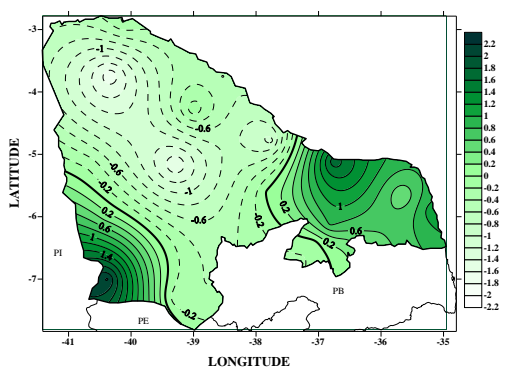

(a)

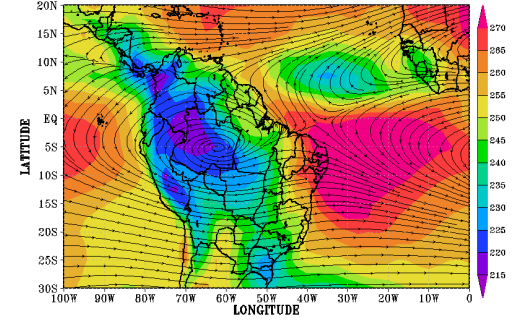

(b) 
Figura 2 - (a) Padrão espacial do segundo fator temporal da velocidade do vento e (b) climatologia semestral (março a agosto) de radiação de ondas longas $\left(\mathrm{Wm}^{-2}\right)$ e vento em 200 $\mathrm{hPa}$ (linhas de corrente, $\mathrm{ms}^{-1}$ ).

\section{CONCLUSÕES}

A partir dos resultados encontrados neste estudo, ao serem definidos os ranges dos critérios de controle de qualidade (diferença absoluta, intervalos de extremos e variações máximas) das velocidades observadas, levar-se-á em consideração a variabilidade espaço-temporal do regime de vento da região, adotando-se valores distintos para cada conjunto de dados, em função da localização da torre anemométrica e período do ano que está sendo analisado.

\section{REFERÊNCIAS}

[1] RICHMAN, M. B. Rotation of principal components. Journal of Climate, 6:293-335, 1986.

[2] ESCOBAR, G. C. J. Padrões sinóticos associados a ondas de frio na cidade de São Paulo. Revista Brasileira de Meteorologia, 22(2):241-254, 2007.

[3] OKOOLA, R. E. Spatial evolutions of the active convective patterns across the Equatorial Eastern Africa region during northern hemisphere spring season using Outgoing Longwave Radiation records. Meteorology and Atmospheric Physics, 66(1):51-63, 1998. 